The overall mean number of comorbidities was 3 (range of $0-7)$ with a mean of 3.5 for those with an incorrect code. Most common co-morbidities were hypertension (30\%), type two diabetes (22\%), ischaemic heart disease/heart failure (17\%), atrial fibrillation (17\%), and other malignancy (15\%).

Conclusions Recommendations to improve clinical coding included: identifying clinical and administrative points during patient journey to review ICD 10 diagnostic code; use of multiple diagnostic codes; and staff education around data collection. The difficulties identified reflect the challenge of accurate clinical coding within the context of an increasingly complex caseload and multiple co-morbidities. It highlights the importance of developing more nuanced approaches to clinical coding within the palliative care setting.

\section{OUR JOURNEY TO IMPLEMENTING THE CASTLE REGISTER: THE LOCAL ELECTRONIC PALLIATIVE CARE COORDINATION SYSTEM (EPACCS) FOR COVENTRY AND WARWICKSHIRE}

Sarah MacLaran, Monica France, Claire Magee, Kay Greene, Hazel Blanchard, Kate Day. UHCW NHS Trust, The Myton Hospices, NHS Arden and Greater East Midlands Commissioning Support Unit, Coventry and Warwickshire Partnership NHS Trust, Mary Ann Evans Hospice, South Warwickshire CCG, Coventry and Rugby CCG

\subsection{6/bmjspcare-2019-ASP.136}

Background EPaCCS enable the recording and sharing of key details and care preferences for patients approaching the end of life, supporting co-ordination of care and delivery of the right care, in the right place, by the right person, at the right time. Core content was identified in an NHS England national information standard for end of life care co-ordination (ISB 1580).

Methods In 2011 a local EPaCCS implementation group was formed with clinical and IT representation from 3 CCGs, 3 acute NHS Trusts, 2 community NHS Trusts, 4 independent hospices, GPs, out-of-hours providers and the ambulance service. From 2012-2014 existing EPaCCS across the country were viewed, an appropriate IT platform was identified, clinical staff were involved to agree content, and bespoke clinical templates were built. From 2014-2016 development took place through a test-launch-review cycle. Information governance issues were addressed with an executive governance policy, standard operating procedure and data sharing agreements. Extensive communication supported a launch across providers.

Results The system went live in November 2016. Over 600 patients have a CASTLE Register record and over 900 staff have been trained and licensed to use the system. It can be viewed in acute, community and hospice settings with automated notifications sent to ambulance and out-of-hours services. Key challenges have included the need for continuous collaboration between all providers; clinical/IT leads learning to speak a common language; interoperability between 10 clinical IT systems; need for culture change across the entire health economy.

Conclusions The journey through engagement, scoping, development and communication has been challenging with many obstacles to overcome along the way. Throughout, the patient has been kept at the centre with passionate clinical leadership and perseverance over years. Ongoing sustainability is being supported through engagement of local clinical champions, CCG funding incentives for GPs, shared resources, training and video learning.

\section{WHERE CHILDREN DIE; A RETROSPECTIVE COHORT STUDY OF CHILD DEATH OVERVIEW PANEL (CDOP) DATA}

Davina Hartley, Kate Renton, Catriona McKeating, Liz Lyles, Louise Clarkson, Anton Mayer. Yorkshire and Humber Children's Palliative Care Network, Sheffield Children's Hospital, Bradford Teaching Hospitals Trust, Forget Me Not Children Hospice, Bradford Safeguarding Children Board, Sheffield Children Hospital

10.1136/bmjspcare-2019-ASP.137

Aims There is a statutory requirement to review all child deaths as part of the safeguarding children guidance. The aim of this cohort study is to collate and evaluate child death data from all Child Death Overview Panels within the Yorkshire and Humber region to inform strategic planning, justify funding, and ultimately improve the care provided to children.

Methods Retrospective cohort study. Data audited from all 14 CDOPs in the region. Descriptive analysis performed using SPSS. The dataset included: 3 full years of CDOP data (2013/ 2014 - 2015/16), all child deaths (expected and unexpected), age (0-17 inclusive), gender, ethnicity, partial postcode, place of death and category of death following review. Ethical approval not required as audit and no patient identifiable data collected. Missing data excluded from analysis on a pairwise basis.

Results Over the study period there were 1221 deaths, with an average mortality rate of 407 deaths pa (total child population=1.1 million, 3.5 deaths $/ 10000$ children). The major causes of death were perinatal/neonatal events, and chromosomal, genetic and congenital anomalies; Combined these account for $60 \%(\mathrm{n}=712 / 1183)$ of the dataset. 'Expected' deaths accounted for 64\% $(n=730 / 1149)$ of all child deaths. Place of death for expected deaths $=$ Hospital deaths: $77 \%$ $(n=564)$ Home: 9\% $(n=67)$, Hospice: 13\% $(n=91)$. When ethnicity data analysed for place of death (excluding neonatal and unexpected deaths), a greater proportion of white British children $(n=201)$ died at home $(16 \%, n=33)$ or within a hospice $(23 \%, n=47)$ when compared to Asian children (Home: 12\%, $\mathrm{n}=14$; Hospice: 9\%, $\mathrm{n}=10$ ); Chi-squared 15.07, $\mathrm{p}=0.002$.

Conclusions

- Place of Death (POD) key quality indicator for EOL care. Limited evidence preferred POD in paediatrics.

- Ethnicity appears to affects POD.

- Oncology patients are more likely than children dying from other causes to die at home. This is likely due to the provision of POON services.

115 EXPERIENCE OF USING INTRAVENOUS ANTIBIOTICS IN AN INPATIENT HOSPICE UNIT

Sarah Sneller, Bethany Wright. St Peter's Hospice (Bristol)

\subsection{6/bmjspcare-2019-ASP.138}

Background The rationale for initiating intravenous antibiotics is variable (symptom control vs life prolongation). There is limited data to show efficacy. Not all hospice inpatient units 